\section{Application of the micronucleus assay performed by different scorers in case of large-scale radiation accidents}

\author{
Kamila Rawojé, \\ Dorota M. Tarnawska, \\ Justyna U. Miszczyk, \\ Jan Swakoń, \\ Liliana Stolarczyk, \\ Marzena Rydygier
}

\begin{abstract}
$\overline{\text { Abstract. Mass casualty scenarios of radiation exposure require high throughput biological dosimetry techniques }}$ for population triage, in order to rapidly identify individuals, who require clinical treatment. Accurate dose estimates can be made by biological dosimetry, to predict the acute radiation syndrome (ARS) within days after a radiation accident or a malicious act involving radiation. Timely information on dose is important for the medical management of acutely irradiated persons [1]. The aim of the study was to evaluate the usefulness of the micronuclei (MNi) scoring procedure in an experimental mode, where 500 binucleated cells were analyzed in different exposure dose ranges. Whole-body exposure was simulated in an in vitro experiment by irradiating whole blood collected from one healthy donor with $60 \mathrm{MeV}$ protons and $250 \mathrm{keV}$ X-rays, in the dose range of 0.3-4.0 Gy. For achieving meaningful results, sample scoring was performed by three independent persons, who followed guidelines described in detail by Fenech et al. [2, 3]. Compared results revealed no significant differences between scorers, which has important meaning in reducing the analysis time. Moreover, presented data based on 500 cells distribution, show that there are significant differences between MNi yields after 1.0 Gy exposure of blood for both protons and X-rays, implicating this experimental mode as appropriate for the distinction between high and low dose-exposed individuals, which allows early classification of exposed victims into clinically relevant subgroups.
\end{abstract}

Key words: biological dosimetry $\bullet$ cytokinesis-blocked micronucleus assay $\bullet$ frequency of micronuclei

K. Rawojć ${ }^{\bowtie}$, D. M. Tarnawska

Faculty of Physics, Astronomy and Applied

Computer Science,

Jagiellonian University,

11 Łojasiewicza Str., 30-348 Krakow, Poland,

Tel.: +48 883740081 ,

E-mail: kamila.rawojc@uj.edu.pl

J. U. Miszczyk

Department of Experimental Physics

of Complex Systems,

The Henryk Niewodniczański Institute of Nuclear

Physics of the Polish Academy of Sciences,

152 Radzikowskiego Str., 31-342 Krakow, Poland

J. Swakoń, L. Stolarczyk, M. Rydygier

Cyclotron Center Bronowice,

Proton Radiotherapy Group,

The Henryk Niewodniczański Institute of Nuclear

Physics of the Polish Academy of Sciences,

152 Radzikowskiego Str., 31-342, Krakow, Poland

Received: 24 September 2014

Accepted: 20 May 2015

\section{Introduction}

The possibility to determine received radiation dose and the assessment of risk to injury is one of the key tasks of biological dosimetry, important not only to the people occupationally exposed to ionizing radiation, but to those of the general population as well. Individual dose assessment in clinical management of exposed subjects is crucial, if it comes to large-scale radiation accidents, when the number of victims reaches hundreds. It is important to rapidly distinguish the severely exposed individuals, who require early medical treatment, from those less exposed or unexposed.

Among the biodosimetry tools, the micronucleus (MN) test in human peripheral lymphocytes is one of standardized cytogenetic technique recommended by International Atomic Energy Agency (IAEA), as an alternative to the dicentric (DC) test for estimating effects of accidental overexposures [3, 4]. The micronuclei (MNi) are chromatin particles formed in cytoplasm, which originate from acentric fragments or whole chromosomes. Many studies have shown that the number of radiation induced $\mathrm{MNi}$ is correlated with radiation dose and quality [4]. 
For retrospective biological dosimetry purposes, the MNi frequency evaluated in peripheral blood lymphocytes drawn from subjects exposed to unknown radiation doses is converted to absorbed dose by reference to a dose-response calibration curve [5]. Since the number of potentially exposed individuals in large scale radiation accident is high, triage and medical management require a fast and reliable dose assessment [5]. The MN assay has been proposed as an alternative to the $\mathrm{DC}$, as MNi scoring is relatively simple and less time consuming $[5,6]$. To enhance the capacity of cytogenetic biodosimetry in case of emergency, three main strategies were proposed. Firstly, a triage mode with a reduced number of binucleated $(\mathrm{BN})$ cells scored by visual counting. Secondly, the establishment of international laboratory network and thirdly, the automation of the scoring $[7,8]$. The very recent paper showed a triage version for MN assay, where only $200 \mathrm{BN}$ cells per subject were proposed to be scored for detecting absorbed radiation doses [7, 8].

In the case of a large event, for example, Fukushima [4], Chernobyl [4], or a 'dirty bomb', there may be problems with infrastructure and power outages, as well as wide-spread panic, that stops emergency personnel from getting to the place where they are needed. There is also a risk that the samples might not be collected under the controlled conditions or sample tracking and processing may be delayed [9]. Yet, there are no reported studies about possibilities to score MNi by people without any cytogenetic experience, which might enhance the process of dose assessment in case of radiation accidents.

The aim of presented study was to evaluate the usefulness of MN assay in a cytogenetic experiment, where only $500 \mathrm{BN}$ cells, instead of 1000 were analyzed by three independent persons, which allows screening of many individuals within a shorter time interval. Another objective of this study was to examine the consistency of the results obtained by three scorers, where only one had a previous experience in performing MN assay. Throughout the whole experiment scorers did not have an opportunity to consult their results.

Whole-body exposures were simulated in an in vitro experiment by irradiating whole blood. Dose response calibration curves for the measuring points in the dose range $0.3-4.0 \mathrm{~Gy}$ of $60 \mathrm{MeV}$ protons and $250 \mathrm{keV}$ X-rays for one healthy donor, derived by following standardized criteria, were compared with those obtained in presented experimental mode. Point $0.0 \mathrm{~Gy}$ was also included in analysis in order to detect MNi background for examined individual.

\section{Materials and methods}

\section{Radiation exposure}

Whole blood was drawn from one healthy, non-smoking donor (female, age 40 years) into heparinized syringes by phlebotomy and immediately divided into aliquots of $1.5 \mathrm{ml}$. Blood samples were irradiated at the H. Niewodniczański Institute of
Nuclear Physics of the Polish Academy of Sciences, Kraków, Poland (IFJ PAN) with a $60 \mathrm{MeV}$ protons and $250 \mathrm{keV}$ X-rays separately. The Human Bioethical Committee of the Regional Medical Board in Kraków, Poland approved the informed consent form used in this study (No. 124/KBL/OIL/2013).

\section{The dosimetry of the proton beam}

The AIC-144 isochronous cyclotron, designed at the IFJ PAN was recently adapted to medical applications and used in this study for proton irradiation experiments. The dosimetry of the proton beam was accomplished by using the PTW UNIDOS T10001 instrument and the semi-flex ion chamber, PTW TM31010. The proton beam intensity was controlled by two transmission PTW ionization chambers, type TM7862, connected to the electrometers. During irradiation the TM7862 ionization chambers carried out the function of dose monitors. The time and spatial stability of the beam were controlled on-line by a sixth sector dedicated ion chamber, which consisted of four ion chambers in the form of quarters, a circle ion chamber and a ring one.

The dose measurements were performed in the middle of spread out Bragg peak, using a solid phantom (PMMA - poly (methyl methacrylate)). The experiments were performed with a 40-mm diameter collimated beam. Overall uncertainty of dosimetry was about $3 \%$, the precision of dose delivery was better than $0.5 \%$. The average dose rate of the proton beam during irradiation was $0.075 \mathrm{~Gy} / \mathrm{s}$. Dosimetric equipment was calibrated at the IFJ PAN, according to the TRS 398 protocol [10]. The therapeutic ${ }^{60}$ Co unit - Theratron 780 E with the PTW Farmer ionization chamber type TM30010, a water phantom and the UNIDOS webline T10021 were used during calibration.

\section{Proton and X-ray irradiation}

For dose-response studies, in the range of $0.3-4.0 \mathrm{~Gy}$ (chosen doses were as follows 0.3, 0.5, 0.75, 1.0, 1.5, 2.0, 2.5, 3.0 and 4.0 Gy), heparinized whole blood samples, were irradiated in Eppendorf vials of $2 \mathrm{~cm}$ long, set in a specially designed PMMA phantom located at the isocenter, at a distance of $93 \mathrm{~mm}$ from the final collimator. The average dose rate used was $0.15 \mathrm{~Gy} / \mathrm{s}$. Immediately after irradiation the vials with irradiated blood underwent the cytogenetic culturing procedures.

To collect more data, all blood samples were irradiated in the same dose ranges, using a Phillips X-ray machine (model MCN 323, $250 \mathrm{keV}, 10 \mathrm{~mA}$ and a dose rate of $1.00 \mathrm{~Gy} / \mathrm{min})$. The vials were held in a polyethylene box. The dimensions of the radiation field were $20 \times 20 \mathrm{~cm}^{2}$ and the source to the surface distance was $34.8 \mathrm{~cm}$. After irradiation the tubes with blood were transferred to a box containing ice water and transported to the laboratory for cell culture straight away. Prior to irradiation of cells, the X-ray dose was measured by using the same ion chamber as for the 
proton beam dosimetry. Both proton and X-ray irradiations were carried out at room temperature $\left(20^{\circ} \mathrm{C}\right)$. Unexposed samples served as control (0.0 Gy).

\section{Cultivation of human lymphocytes}

The culture technique was fully described by Fenech et al. [11, 12]. In brief, cultures were set up with $0.5 \mathrm{ml}$ whole blood added to $4.5 \mathrm{ml}$ of RPMI 1640 culture medium (PAA Laboratories $\mathrm{GmbH}$, Pasching, Austria), supplemented with $10 \%$ heat-inactivated fetal calf serum (Gibco, Carlsbad, U.S.A.), $2 \mathrm{mM}$ L-glutamine and antibiotics $(100 \mathrm{U} / \mathrm{ml}$ penicillin and $100 \mathrm{~g} / \mathrm{ml}$ streptomycin Polfa Tarchomin, Warsaw, Poland). The lymphocytes were stimulated by the addition of $450 \mu \mathrm{l}$ of phytohemagglutinin (PHA, PAA Laboratories $\mathrm{GmbH}$, Pasching, Austria). The cultures were incubated for $72 \mathrm{~h}$ at $37^{\circ} \mathrm{C}$ at $5 \% \mathrm{CO}_{2}$ in a humidified incubator. After $44 \mathrm{~h}$ of culture time $6 \mu \mathrm{g} / \mathrm{ml}$ cytocholasin-B in dimethylsulfoxide (Sigma-Aldrich, St. Louis, U.S.A.) was added to block the cytokinesis. When $72 \mathrm{~h}$ of incubation passed, the cultures were harvested by centrifuging, re-suspended in a hypotonic solution of $0.075 \mathrm{M} \mathrm{KCl}$ and fixed with methanol:acetic acid $(3: 1)$. Cells were three times washed with fixative, than dropped onto clean microscope slides, air-dried and stained with 4\% Giemsa. Prepared samples were stored in room temperature until the examination.

\section{Aberration scoring}

The slides were analyzed for the number of micronuclei in (MNi frequency) BN cells according to the criteria described by Fenech [2]. Chromatin bodies that are round-shaped, similar in staining characteristics and smaller than one-third the size of the main nucleus, not re-fractile and not connected to the main nucleus were counted as micronuclei [11]. All slides were coded and blinded to the scorers by a chosen individual. Each slide was scored by three independent persons manually, at $400 \times$ magnification using the same model of Nikon light microscopes.

\section{Statistical methods}

The data analysis was performed using the Microsoft Office Excel 2010 program and the MNi frequency, as well as dose-response curves were obtained by the OriginPro 9.032 bit (OriginLab Co., Northampton, MA, U.S.A.). A curve fitting by linear-quadratic and cubic models was done by least square regression method and the goodness of fit was tested by Chi-squared test.

For each sample the MNi frequency (the ratio of the number of $\mathrm{MNi}$ and the chosen number of $\mathrm{BN}$ cells, multiplied by one hundred), given in percent, was assessed based on analysis of 500 and $1000 \mathrm{BN}$ cells. The error given in each measurement point is the standard error (SD) of the mean. To investigate if the results obtained by three scorers are comparable, Pearsons' correlation coefficient was calculated. For further understanding the mechanism of MNi formation, the relative biological effectiveness (RBE) was derived as a ratio of MNi frequency induced by protons relative to X-rays.

\section{Results}

\section{Frequency of micronuclei in experimental mode}

For experimental purposes, the analysis of 500 and 1000 binucleated cells was performed. Figures 1 and 2, show a comparison between the results derived by scoring 500 vs. $1000 \mathrm{BN}$ cells induced by $250 \mathrm{keV}$ X-rays or $60 \mathrm{MeV}$ protons. The measurement points represent the arithmetic mean of MNi frequency obtained by three different scorers. The error bars are shown as SD of the means with the $95 \%$ confidence limits.

The coefficients of the best fitting polynomials are given in Tables 1 and 2, for both linear-quadratic and cubic models for X-rays and protons, respectively. Because of the overlapping of the curves in Fig. 1 only coefficients for $500 \mathrm{BN}$ cells are shown in Table 1 . The results showed that studied beams have different effect on the MNi frequency.

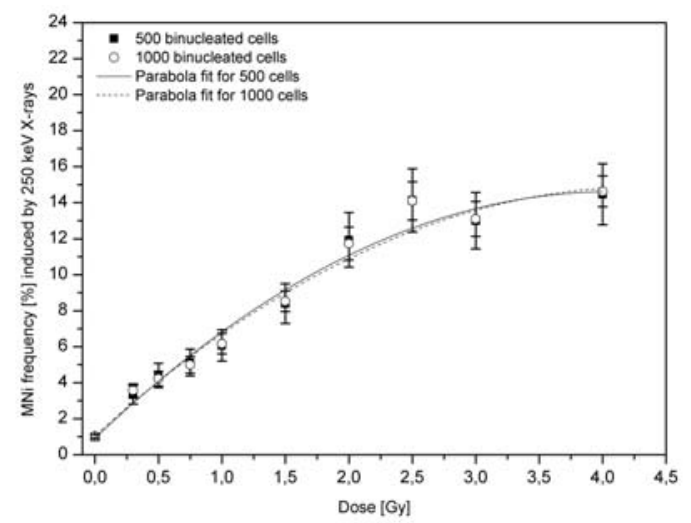

Fig. 1. The frequency of MNi induced by $250 \mathrm{keV}$ X-rays and obtained by averaging the results derived for the donor by three independent scorers. The error bars represent SD of the means and indicate $95 \%$ confidence limits.

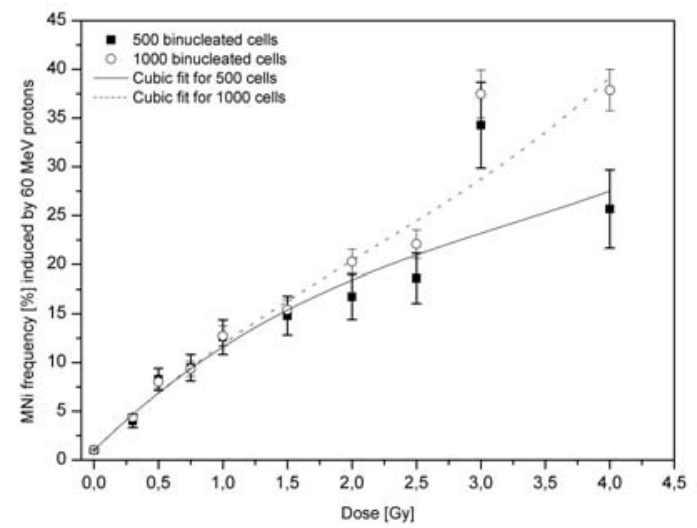

Fig. 2. The frequency of MNi induced by $60 \mathrm{MeV}$ protons and derived by averaging the results for the donor by three independent scorers. The error bars indicate 95\% confidence limits and represent SD of the means. 
Table 1. The coefficients of MNi frequency fits for the parabola model (X-rays) obtained for $500 \mathrm{BN}$ cells

\begin{tabular}{|c|c|c|}
\hline Model & \multicolumn{2}{|c|}{500 binucleated cells } \\
\hline Equation & \multicolumn{2}{|c|}{$y=A+B^{*} x+C^{*} x^{\wedge} 2$} \\
\hline Reduced Chi-square & \multicolumn{2}{|c|}{1.70316} \\
\hline Adj. R-square & \multicolumn{2}{|c|}{0.9843} \\
\hline & Value & Standard error \\
\hline$\overline{\mathrm{A}}$ & 0.91697 & 0.11745 \\
\hline B & 6.73589 & 0.46867 \\
\hline C & -0.82847 & 0.15099 \\
\hline
\end{tabular}

The response of lymphocytes presented as MNi frequency was higher for protons than X-rays for both scenarios (where 500 or 1000 cells were scored) in whole dose range. Moreover, for both protons and X-rays, the ratio of MNi steeply increases with increasing radiation dose, in particular, whereby it is possible to discriminate doses below and above $1.0 \mathrm{~Gy}$ using this endpoint in case of each studied types of radiation.

Presented results revealed no statistically significant differences in studied dose range $(0.0-4.0 \mathrm{~Gy})$ in cells exposed to $250 \mathrm{keV}$ X-rays and 0.0-3.0 Gy for protons. Only for 4.0 Gy of protons a statistically significant difference was observed between analyzed 500 vs. 1000 BN cells. Additionally, slightly higher measurement errors in dose $3.0 \mathrm{~Gy}(34.27 \pm$ 0.34 for $500 \mathrm{BN}$ cells vs. $37.46 \pm 0.35$ for $1000 \mathrm{BN}$ cells) were observed in Fig. 2.

The Pearson correlation coefficient derived by comparing results of scoring 500 vs. $1000 \mathrm{BN}$ cells was $0.99(p<0.05)$ for X-rays and $0.97(p<0.05)$ for protons, suggesting that the results are strongly correlated. Based on obtained results the mean value of RBE for protons was $1.69 \pm 0.56$ for $500 \mathrm{BN}$ cells and $1.86 \pm 0.48$, when $1000 \mathrm{BN}$ cells were analyzed. These RBE values are pointed in the error margin.

\section{Intra-comparison and validity of results based on scoring 1000 BN cells}

To examine the consistency of the results obtained by three independent scorers (scorer 1 and 2 had no previous experience in performing $\mathrm{MN}$ assay) an intra-comparison was performed. This evaluation was done for scored $1000 \mathrm{BN}$ cells. Figures 3 and 4 show results of analysis of each single scorer

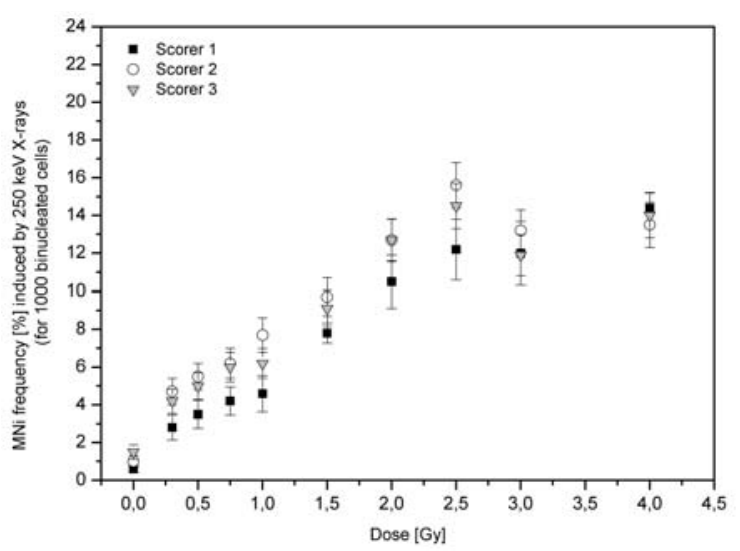

Fig. 3. The intra-comparison of the results obtained by three independent scorers, calculated for $1000 \mathrm{BN}$ cells irradiated with X-rays. Only scorer 3 had previous experience in performing MN assay.

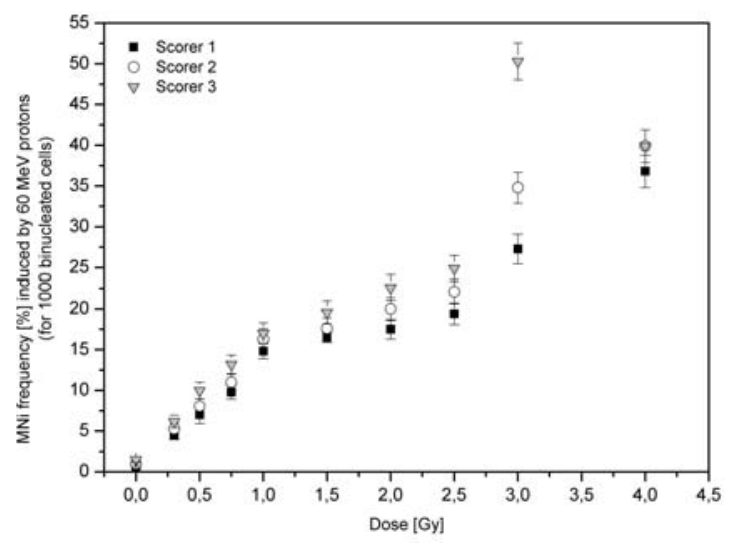

Fig. 4. The intra-comparison of the results derived by three independent persons for $1000 \mathrm{BN}$ cells irradiated with protons. Scorer 1 and scorer 2 had no experience in MNi scoring.

in prepared experimental mode for lymphocytes irradiated by X-rays or protons.

In both studied cases (Figs. 3 and 4), the results revealed no significant difference and a matching correlation between scorers. However, it is visible that inexperienced scorer 1 counts less $\mathrm{MNi}$ than scorers 2 and 3 . Whereas, inexperienced scorer 2 , scores more $\mathrm{MNi}$, compared to results of remainder scorers. Only at the dose of $3.0 \mathrm{~Gy}$ in case of cells irradiated by protons a discrepancy in results was observed, which may explain the reason of increased error bars in Fig. 2 at the similar dose. The difference in obtained results in this measurement point

Table 2. The coefficients of MNi frequency fits for the cubic model (protons) derived for $500 \mathrm{BN}$ cells and $1000 \mathrm{BN}$ cells

\begin{tabular}{|c|c|c|c|c|}
\hline Model & \multicolumn{2}{|c|}{500 binucleated cells } & \multicolumn{2}{|c|}{1000 binucleated cells } \\
\hline Equation & \multicolumn{2}{|c|}{$y=A+B * x+C^{*} x^{\wedge} 2+D^{*} x^{\wedge} 3$} & \multicolumn{2}{|c|}{$y=A+B^{*} x+C^{*} x^{\wedge} 2+D^{*} x^{\wedge} 3$} \\
\hline Reduced Chi-square & \multicolumn{2}{|c|}{1.81192} & \multicolumn{2}{|c|}{3.40968} \\
\hline \multirow[t]{2}{*}{ Adj. R-square } & \multicolumn{2}{|c|}{0.9528} & \multicolumn{2}{|c|}{0.9794} \\
\hline & Value & Standard error & Value & Standard error \\
\hline$\overline{\mathrm{A}}$ & 0.98852 & 0.25565 & 1.02894 & 0.16896 \\
\hline B & 13.04832 & 2.47545 & 12.79884 & 1.91914 \\
\hline $\mathrm{C}$ & -2.726 & 2.29425 & -2.30541 & 1.77288 \\
\hline $\mathrm{D}$ & 0.28027 & 0.47465 & 0.37220 & 0.36211 \\
\hline
\end{tabular}


has been reanalyzed three times by each scorer. Nevertheless, the results remained similar and again a discrepancy was noticed. The same trends were observed when $500 \mathrm{BN}$ cells were analyzed (data not shown). The mean value of Pearson correlation coefficient calculated for scorers, when $1000 \mathrm{BN}$ cells are analyzed was the same for X-rays $(0.95$, $p<0.05)$ and for protons $(0.95, p<0.05)$.

\section{Discussion}

Management of large-scale radiation accidents requires a high throughput biological dosimetry technique for population triage, where rapid identification of wounded persons is most urgent. For triage purposes several approaches might be considered. Clinical symptoms are useful in estimating radiation effects based on an organ level, while the assessment of radiation-induced effects upon cytogenetic tests presents a different approach. Naturally, each system has its benefits and limits, with respect to the usefulness for specific applications. The micronucleus assay is a well standardized and validated technique for in vivo evaluation of radiation exposure, which is used as an alternative to the dicentric chromosomes assay (DCA) $[4,5]$. Due to the necessity of specialized expertise, devices or facilities, to which availability is limited, dicentric chromosome test has certain constrains $[4,5]$. During last years, several improvements of MN assay have been achieved, in order to make it suitable for simple and rapid analysis in case of radiation emergency [5]. There have been formed many algorithms to expedite $\mathrm{MN}$ image analysis in automated scoring procedure, but because of its costs and access limitations, laboratories are searching for a less constrained strategy of scoring based on manual analysis of reduced number of binucleated cells.

Concerning the usefulness of the method in reasonable therapeutic decision making, the goal of this study was to verify the MNi scoring procedure in experimental mode, where only $500 \mathrm{BN}$ cells, instead of 1000 were analyzed in a different dose ranges. Another study objective was to verify if persons without experience in performing MN assay, may analyze samples as well as an experienced person, which would speed up sample screening to clinically relevant subgroups of potentially overexposed individuals. In order to determine MN assay's capability of distinguishing low from high-dose exposed, blood samples collected from one healthy donor have been irradiated in dose range 0.3-4.0 Gy using X-rays or protons. The experiment was designed in this way, to mimic the real scenario of a radiological event, that is, a radiation accident, which involves numerous overexposed individuals.

Presented results showed no significant difference in scoring $500 \mathrm{BN}$ cells, instead of 1000 in whole dose range for X-rays and in 0.3-3.0 Gy for protons. Only for $4.0 \mathrm{~Gy}$ of protons statistically significant difference was observed. Starting from dose $1.5 \mathrm{~Gy}$ all measurement points had lower value, when only $500 \mathrm{BN}$ cells were scored in case of protons. Observed effects in our studies may be explained by a difference in the mechanism of damage induction by X-rays and protons. At higher doses proton beams produce dense ionization tracks resulting from spatially localized energy deposition in 'sequential bursts'. The dose dense energy deposition results in an increase in locally multiply damaged sites (LMDS) or 'clustered DNA damage', which increases with linear energy transfer (LET) and dose [13]. It is probable these effects, presented as a frequency of $\mathrm{MNi}$ (as BN cells with one MN, two, three MNi) are better seen, where $1000 \mathrm{BN}$ cells are analyzed.

The number of BN cells scored in the MNi analysis in triage mode is still under investigation [5]. Our approach to establish a different triage mode for MNi scoring is based on the statistical properties of the Poisson distribution. One of the properties of the Poisson distribution is that its variance is equal to its expected value. In consequence the accuracy in the estimation of the rate of occurrence of a Poisson event depends only on the number of events. In our case, the MNi scoring precision of MNi determination depends on the number of BN cells with MNi evaluated and not on the total number of BN cells scored [5].

The standardized IAEA protocol [3, 12] recommends scoring $1000 \mathrm{BN}$ cells minimum, however presented results are in agreement with McNamee et al. observations [14]. In McNamee's triage version only $200 \mathrm{BN}$ cells per subject were analyzed and happen to be enough for detection doses $>1.0$ Gy of gamma radiation [14]. Our results confirmed McNamee's observation in whole dose range 0.3-4.0 Gy for X-rays and 0.3-3.0 Gy for protons. Observations done by McNamee's seem to be explained by relatively uniform distribution of energy with gamma rays in comparison to the 'sporadic' or non-uniform distribution of energy among cells with protons. The triage approach was also implemented in other cytogenetic test, for example, the dicentric chromosome assay as initial screening of subjects in case of radiation emergency $[15,16]$. Analysis of 50 metaphase spreads in DCA, instead of routinely 500 or 1000 , increases the threshold level of detection 1.0-2.0 Gy, which is still adequate to provide treatment of the ARS $[15,16]$.

Between May 2010 and April 2013, a collaborative project within Framework Programme 7 of the European Union MULTIBIODOSE, was tasked with the aim of establishing a fully functional and ready to respond in case of a mass casualty situation biodosimetric network and succeed [6]. In the MULTIBIODOSE project, three triage categories were presented as: 'low dose' category with doses of $1.0 \mathrm{~Gy}$, requiring no direct treatment, then a 'medium dose' category with doses ranging from 1.0 to $2.0 \mathrm{~Gy}$, for which medical follow-up or treatment is necessary and a class of a 'high dose' with doses exceeding 2.0 Gy requiring urgent medical intervention [6]. In our studies the laboratory staff was able not only to stratify of radiation-exposed individuals according with these rules, but to identify each physical dose for X-rays and for 0.3-3.0 Gy for protons.

To examine the consistency of the results obtained by three independent scorers (of whom only one: 
scorer 3 had previous experience in performing cytogenetic tests) an intra-comparison of MNi frequency was performed, which was shown in Figs. 3 and 4. Observation based on obtained results revealed matching correlation (the mean value of the Pearson correlation coefficient was: $0.95, p<0.05$ ) for all scorers in whole dose ranges for both X-rays and protons (Figs. 3 and 4). Higher dispersion in results of one measuring point of $3.0 \mathrm{~Gy}$ was detected in frequency of MNi induced by protons (Fig. 4), but yet shown values still indicate high dose estimation accuracy in both scoring modes. Presented practice confirms that MN assay requires less expertise in scoring compared to DCA. The triage mode of MN assay appears to be a sensitive and reliable approach in large-scale radiation accidents. Furthermore, agreement of the results obtained by three independent scorers encourages a mutual cooperation to prepare a validated mode for biodosimetry in case of emergency.

The biological responses of irradiated cells are also dependent on the dose rate [17]. Due to technical limitations of the dose delivery, we were not able to deliver protons and photons at similar dose rates, therefore, a direct comparison of the dose rate effects in our model is difficult. However, the observed differences in effects from proton vs. X-rays irradiations are likely to have been influenced by the differences in dose rates, to some extent, but we believe the differences in energy spectrum are the primary reason for the observed difference to a large extent.

The dose-response relationships of chromosome aberration frequencies induced by the proton beam irradiation have been reported in the literature, yet the frequencies have been found to be dependent on proton energy [18]. The radiation effects were influenced by the sites in which the cells were irradiated [19]. However, there was no reported comparison between X-rays and protons response curves.

Despite the differences, our results are in good agreement with those reported by others. The observed variation can be explained by additional factors starting on the culture conditions, number of scored cells, scoring criteria and ending on the difference in experience of the scorers $[20,21]$. Furthermore, this comparison justified the recommendations of preparing own dose-response curve by every laboratory, so that, in the case of overexposure all conditions are repetitive [22].

The RBE for protons is equal to the ratio of the dose of a reference radiation (X-rays) to that of protons necessary to produce the same biological effect. The RBE depends on the reference radiation, dose, dose rate, biological system and the biological endpoint being analyzed. In our study the mean value of RBE for protons was presented (1.69 \pm 0.56 for $500 \mathrm{BN}$ cells and $1.86 \pm 0.48$ for $1000 \mathrm{BN}$ cells). For therapeutic proton beams $(60-260 \mathrm{MeV})$ across various doses, the RBE is about 1.1-1.2 (Fig. 4) in the centre of the spread out Bragg peak (SOBP, Fig. 2) [23-25]. The reported values for the RBE of a proton beam are ranging from 0.8 to 2.0 [19]. However, in the presented study, the dose and depth at which the blood was irradiated were found to have a considerable influence upon the RBE values of the proton beam. These will be considered in the future work in order to determine the RBE of protons by cytogenetic abnormalities (CA), fluorescent in situ hybridization (FISH) and premature chromosome condensation (PCC) techniques.

Given the complex nature of radiation, it is unlikely that any single biodosimetry assay can be used as a one dedicated tool [26]. Physical dosimetry may not be available for a number of hours after an incident and clinical signs are not necessarily accurate [27]. It seems that, in the event of a radiological catastrophe, immediate triage would be accomplished through a combination of physical dosimetry, history of an individual's location, clinical signs and symptoms and individual hematology assessment, with other methods such as the MN assay used for long-term risk assessment [27].

Overall, first results showed proposed strategies as promising in improving performance of MN assay as a biodosimeter in mass casualties, allowing to reduce the scoring time to improve the accuracy of evaluation. This study shows that analyzing $500 \mathrm{BN}$ cells gives reliable and accurate individual dose estimation over whole, examined dose range $0.3-4.0 \mathrm{~Gy}$ for $\mathrm{X}$-rays and 0.3-3.0 Gy for protons as a first approach in large scale radiological accidents. In the near future, the laboratory is planning to improve triage mode and expand the dose range up to $5.0 \mathrm{~Gy}$. Moreover, the laboratory is carrying out further research using PCC assay, in order to define even more significant cut-off levels for quick classification of overexposed persons in medical treatment categories. Further studies for more donors to identify potential differences in individual sensitivity are also required.

Acknowledgments. The authors would like to thank Mrs. Jolanta Adamczyk for her assistance in laboratory procedures. Second, authors gratefully acknowledge the donors for providing blood samples and also technical staff responsible for the irradiation and dosimetry. These investigations were conducted as a part of an extended examination of $60 \mathrm{MeV}$ protons from AIC-144 isochronous cyclotron, at the Institute of Nuclear Physics in Krakow, Poland (IFJ PAN) by cytogenetic and molecular methods and were partially supported by grant DEC-2013/09/D/NZ7/00324 from the National Science Centre, Poland.

\section{References}

1. Van del Kogel, A., \& Joiner, M. (2009). Basic clinical radiobiology. United Kingdom: Hodder Education.

2. Fenech, M., Holland, N., Chang, W. P., Zeiger, E., \& Bonassi, S. (2003). HUMN project: detailed description of the scoring criteria for the cytokinesis-block micronucleus assay using isolated human lymphocyte cultures. Mutat. Res., 534, 65-75.

3. IAEA. (2001). Cytogenetic analysis for radiation dose assessment. A manual. Vienna: International Atomic Energy Agency. (Technical Reports Series no. 405).

4. Vral, A., Fenech, M., \& Thierens, H. (2011). The micronucleus assay as a biological dosimeter of in vivo ionising radiation exposure. Mutagenesis, 26(1), 11-17. 
5. Bolognesi, C., Ropolo, M., Roggieri, P., \& Bruzzi, P. (2014). Biological dosimetry by the micronucleus test: A validation study for the application in radiation mass casualties. Retrieved September 14, 2014, from CSO database on the World Wide Web: https://www.cso.nato.int/pubs/rdp.asp?RDP=STOMP-HFM-223.

6. Thierens, H., Vral, A., Vandevoorde, C., Vandersickel, V., de Gelder, V., Romm, H., Oestreicher, U., Rothkamm, K., Barnard, S., Ainsbury, E., Sommer, S., Beinke, C., \& Wojcik, A. (2014). Is a semi-automated approach indicated in the application of the automated micronucleus assay for triage purposes? Radiat. Prot. Dosim., 159(1/4), 87-94.

7. Bolognesi, C., Balia, C., Roggieri, P., Cardinale, F., Bruzzi, P., Sorcinelli, F., Lista, F., D'Amelio, R., \& Righi, E. (2011). Micronucleus test for radiation biodosimetry in mass casualty events: Evaluation of visual and automated scoring. Radiat. Meas., 46(2), 169-175.

8. Franco, M., Bolognesi, C., De Amicis, A., Amati, A., Di Cristofaro, S., Regalbuto, E., Ropolo, M., Lista, F., \& De Sanctis, S. (2012). Interlaboratory comparison on cytokinesis-block micronucleus assay for X-ray calibration curve and dose prediction in Italy. Effects of Ionizing Radiation Exposure and Countermeasures: Current Status and Future Perspectives S\&T. In T. C. Pellmar (Ed.), Biological effects of ionizing radiation exposure and countermeasures: Current status and future perspectives (paper no. 22). NATO Science and Technology Organization. (STO-MP-HFM-223).

9. Martin, P. R., Berdychevski, R. E., Subramanian, U., Blakely, W. F., \& Prasanna, P. G. S. (2007). Sample tracking in an automated cytogenetic Biodosimetry Laboratory for Radiation Mass Casualties. Radiat. Meas., 42(6/7), 1119-1124.

10. Michalec, B., Swakoń, J., Sowa, U., Ptaszkiewicz, M., Cywicka-Jakiel, T., \& Olko, P. (2010). Proton radiotherapy facility for ocular tumors at the IFJ PAN in Kraków Poland. Appl. Radiat. Isot., 68, 738-742.

11. Fenech, M., Holland, N., Chang, W. P., Zeiger, E., \& Bonassi, S. (1999). The Human MicroNucleus Project - An international collaborative study on the use of the micronucleus technique for measuring DNA damage in humans. Mutat. Res.-Fundam. Mol. Mech. Mutagen., 428(1/2), 271-283.

12. IAEA. (2011). Cytogenetic dosimetry applications in preparedness for and response to radiation emergencies. Vienna: International Atomic Energy Agency.

13. Joksic, G., Pajovic, S. B., Stankovic, M., Pejic, S., Kasapovic, J., Cuttone, G., Calonghi, N., Masotti, L., \& Kanazir, D. T. (2000). Chromosome aberrations, micronuclei, and activity of superoxide dismutases in human lymphocytes after irradiation in vitro. Cell. Mol. Life Sci., 57, 842-850.

14. McNamee, J. P., Flegal, F. N., Greene, H. B., Marro, L., \& Wilkins, R. C. (2009). Validation of the cytokinesis-block micronucleus (CBMN) assay for use as a triage biological dosimetry tool. Radiat. Prot. Dosim., 135, 232-242.

15. Beinke, C., Oestreicher, U., Riecke, A., Kulka, U., Meineke, V., \& Romm, H. (2011). Inter-laboratory comparison to validate the dicentric assay as a cytogenetic triage tool for medical management of radiation accidents. Radiat. Meas., 46, 929-935.

16. Wilkins, R. C., Romm, H., Oestreicher, U., Marro, L., Yoshida, M. A., Suto, Y., \& Prasanna, P. G. (2011). Biological dosimetry by the TriageDicentric Chromosome Assay - further validation of International Networking. Radiat. Meas., 46, 923-928.

17. Konopacka, M., \& Rogoliński, J. (2011). Clastogenic effects in human lymphocytes exposed to low and high dose rate X-ray irradiation and vitamin C. Nukleonika, 56, 253-257.

18. Go, Y. J., Kwon, O. D., Shin, J. H., Kim, S. H., Jeong, K. S., Ryu, S. Y., Park, S. J., Kim, C. H., Kim, T. H., Lee, M. H., Kim, E. J., Kwak, D. M., \& Kang, Ch. M. (2011). Dose estimation with the calibration of dose-response curve of micronucleus in human peripheral lymphocytes induced by $50 \mathrm{MeV}$ proton beams. Iran. J. Radiat. Res. Soc., 8(4), 231-236.

19. Matsubara, S., Ohara, H., Hiroaka, T., Koike, S., Ando, K., Yamaguchi, H., Kuwabara, Y., Hoshina, M., \& Suzuki, S. (1990). Chromosome aberration frequencies produced by a $70-\mathrm{MeV}$ proton beam. Radiat. Res. Soc., 123, 182-191.

20. Fenech, M. (1998). Important variables that influence base-line micronucleus frequency in cytokinesis-blocked lymphocytes - a biomarker for DNA damage in human populations. Mutat. Res., 404, 155-165.

21. Fenech, M. (2000). The in vitro micronucleus technique. Mutat. Res., 455, 81-95.

22. Pajic, J., Rakic, B., Jovicic, D., \& Milovanovic, A. (2014). Construction of dose response calibration curves for dicentrics and micronuclei for $\mathrm{X}$ radiation in a Serbian population. Mutat. Res., 773, 23-28.

23. Pagenetti, H., Niemierko, A., Ancukiewicz, M., Gerweck, L. E., Gotein, M., Loeffler, J. S., \& Suit, H. D. (2002). Relative biological effectiveness (RBE) values for proton beam therapy. Int. J. Radiat. Oncol. Biol. Phys., 53(2), 407-421.

24. Girdhani, S., Sachs, R., \& Hlatky, L. (2013). Biological effects of proton radiation: what we know and don't know. Radiat. Res. Soc., 179, 257-272. Retrieved September 14, 2014 from PubMed database on the World Wide Web: http://www.pubmed.gov. PMID: 23373900

25. Tamizh Selvan, G., Bhavani, M., Vijayalakshmi, J., Solomon, P. F. D., \& Chaudhury, N. K. (2014). Delayed mitogenic stimulation decreases DNA damage assessed by micronucleus assay in human peripheral blood lymphocytes after 60-Co irradiation. DoseResponse, 12(3), 498-508.

26. Sullivan, J. M., Prasanna, P. G., Grace, M. B., Wathen, L. K., Koerner, J. F., \& Coleman, C. N. (2013). Assessment of biodosimetry methods for a mass-casualty radiological incident: Medical response and management considerations. Health Phys., 105(6), 540-554. DOI: 10.1097/HP.0b013e31829cf221.

27. Demidenko, E., Williams, B. B., \& Swartz, H. M. (2009). Radiation dose prediction using data on time to emesis in the case of nuclear terrorism. Radiat. Res., 171, 310-319. 